\title{
EL GÉNERO ELAPHOCERA GENÉ, 1836 (COLEOPTERA, MELOLONTHIDAE, PACHYDEMINAE): REVISIÓN DE LAS SERIES TIPO DE LAS COLECCIONES DEL MUSEO NACIONAL DE CIENCIAS NATURALES (MADRID, ESPAÑA)
}

\author{
I. Sanmartín (*), F. Martín-Piera (*) y M. A. Alonso-Zarazaga (*)
}

\begin{abstract}
Resumen
En este trabajo se designa Melolontha emarginata Gyllenhal, 1817 como la especie tipo de Elaphocerida Reitter, 1902. Se revisan las series típicas de las especies del género Elaphocera Gené, 1836 conservadas en las colecciones de Entomología del Museo Nacional de Ciencias Naturales (Madrid, España). Se designan los lectotipos de Elaphocera (Elaphocerida) ampla Báguena, 1955, Elaphocerida segurensis Escalera, 1923, Elaphocerida pygmaea Escalera, 1923 y Elaphocera (Elaphocerida) torressalai Báguena, 1955. Además, se proporcionan nuevos datos sobre el material típico de las especies Elaphocera pardoi Escalera, 1931, Elaphocera (Elaphocerida) ibicensis Escalera, 1926, Elaphocerida abdelkrimi Escalera, 1934 y Elaphocera baguenae Mancini, 1926, conservado en estas colecciones.

Palabras clave: tipos, MNCN, Elaphocera, Pachydeminae, designación.
\end{abstract}

\begin{abstract}
A review of the type material of genus Elaphocera Gené, 1836 (Coleoptera, Melolonthidae, Pachydeminae) from the collections of Museo Nacional de Ciencias Naturales (Madrid, Spain)
\end{abstract}

Melolontha emarginata Gyllenhal, 1817 is designated as the type species of Elaphocerida Reitter, 1902. The type specimens of the species of genus Elaphocera Gené, 1836, from the entomological collections of Museo Nacional de Ciencias Naturales (Madrid, Spain), are studied. Lectotypes are designated for species Elaphocera (Elaphocerida) ampla Báguena, 1955, Elaphocerida segurensis Escalera, 1923, Elaphocerida pygmaea Escalera, 1923 and Elaphocera (Elaphocerida) torressalai Báguena, 1955. The type material of the following species is also revised: Elaphocera pardoi Escalera, 1931, Elaphocera (Elaphocerida) ibicensis Escalera, 1926, Elaphocerida abdelkrimi Escalera, 1934 y Elaphocera baguenae Mancini, 1926.

Key words: types, MNCN, Elaphocera, Pachydeminae, designation.

\section{Introducción}

El género Elaphocera fue creado por Gené (1836), como parte de una carta dada a conocer por M. Audouin en la sesión del 6 de enero de 1836 de la Sociedad Entomológica de Francia. Este trabajo pasó desapercibido y todos los autores posteriores citan como obra original un trabajo posterior (Gené, 1837b). Este artículo, en forma de separata (Gené, 1837a), se cita por primera vez en la sesión

* Dpto. Biodiversidad y Biología Evolutiva (Entomología). Museo Nacional Ciencias Naturales. José Gutiérrez Abascal, 2; 28006 Madrid. ESPAÑA 
del 5 de Abril de 1837 de la Sociedad Entomológica de Francia. Sin embargo, la publicación formal del trabajo (Gené, 1837b) no se registra hasta la sesión del 5 de julio de 1837 de la Sociedad Francesa. Por tanto, es la comunicación de 1836 la que se debe tomar como referencia de la descripción original del género. El nombre del género deriva de ("elaphos = ciervo / kera= cuerno"), aludiendo a la existencia de una larga espina en el segundo artejo del funículo antenar del macho.

En el mismo año, Dejean (1836) creó el género Leptopus, bajo el que agrupó dos especies: Leptopus denticornis, Dufour nom. nud. (= Elaphocera denticornis Reitter, 1902) y Leptopus bedeau Dufour nom. nud (= Elaphocera bedeaui Erichson, 1840). Este nombre, sin embargo, no está disponible por no tener ninguna especie válida incluida. De todos modos, este nombre ya había sido empleado por Latreille (1809) para designar un grupo de Hemípteros Heterópteros, por lo que Leptopus Dejean queda como sinónimo de Elaphocera Gené, 1836 y homónimo de Leptopus Latreille, 1809. Rambur (1843) señala que Gené propuso el nombre de Elaphocera para corregir este error pero esto no parece posible puesto que Dejean publicó su descripción de Leptopus en Julio de 1836 (Madge, 1988), y Gené la suya de Elaphocera en enero de ese mismo año.

El género Elaphocera Gené ha sido posteriormente objeto de varias monografías que incrementaron considerablemente el número de especies (Rambur, 1843; Burmeister, 1855; Jaquelin du Val, 1860; Kraatz, 1882; Reitter, 1902). Este último dividió el género en dos subgéneros, Elaphocera $s$. str. y Elaphocerida, atendiendo a dos caracteres: la posición de la espina interna de la protibia y la longitud del primer artejo de los metatarsos respecto al segundo. Todos los autores posteriores adoptaron esta subdivisión (Dalla Torre, 1912-1913; Escalera, 1923; Medvedev, 1952; Báguena, 1955, 1967; Baraud, 1966). Posteriormente, Baraud (1975) elevó formalmente el rango taxonómico de los subgéneros de Reitter, aunque ya Escalera (1923) los menciona como géneros independientes. Baraud (1975) propuso considerar al subgénero Elaphocerida Reitter como un género aparte, basándose en la presencia de nuevos caracteres que lo separarían del género Elaphocera s. str., tales como la forma del edeago y el estado alar de las hembras. Sin embargo, el mismo Baraud (1987) señaló la existencia de un error de nomenclatura. Gené (1836) había descrito el género Elaphocera para la única especie Elaphocera obscura, que fue sinonimizada con Melolontha emarginata Gyllenhal, 1817 por Kraatz (1882). Por tanto esta especie debe ser considerada como la especie tipo del género Elaphocera Gené ("especie tipo por monotipia" (ICZN, 1985, art. 68 (d)) y la designación efectuada por Rambur (1843) de Elaphocera bedeaui Erichson, 1840 como la especie-tipo del género no es válida. Baraud (1987) argumenta que al haber incluido Reitter (1902) a E. emarginata (Gyllenhal) dentro del subgénero Elaphocerida, este subgénero caería en sinonimia de Elaphocera Gené. Aunque comúnmente aceptado, el argumento de Baraud, sin embargo, no es válido desde un punto de vista formal. La sinonimia de Elaphocera Gené y Elaphocerida Reitter requeriría haber demostrado la pertenencia al mismo género de las especies-tipo de ambos taxones lo que no es posible porque Reitter no designó especie-tipo para el subgénero Elaphocerida. Para resolver este problema se designa en este trabajo a Melolontha emarginata Gyllenhal como la especie tipo del subgénero Elaphocerida por ser ésta la especie más antigua descrita, incluida en el subgénero de Reitter. De esta forma, puesto que la especie-tipo de Elaphocera Gené, E. obscura, es sinónimo de $E$. emarginata, la especie-tipo de Elaphocerida Reitter, ambos taxones, Elaphocera Gené y Elaphocerida Reitter, pueden considerarse formalmente como sinónimos subjetivos. Por otra parte, las especies del taxon Elaphocera s. str. sensu Reitter fueron incluidas por Baraud en un nuevo género: Ceramida Baraud, 1987. De esta forma la nomenclatura quedó de la siguiente manera:

-Género Elaphocera Gené, 1836 = Leptopus Dejean, 1836 = Elaphocerida Reitter, 1902. Especie-tipo: Melolontha emarginata Gyllenhal, 1817, por designación actual.

-Género Ceramida Baraud, 1987 = Elaphocera sensu Reitter, 1902 (non Gené, 1836). Especie-tipo: Melolontha longitarsis Illiger, 1803, por designación original.

El género Elaphocera Gené, 1836 agrupa actualmente 39 especies distribuidas por todo el área mediterránea (Baraud, 1992). En el Mediterráneo occidental, el género está presente en el Sureste de España, con 20 especies, y, en menor medida, en las Islas Baleares (2 especies), Norte de Africa (2 especies) y Sicilia (1 especie). Los entomólogos españoles Manuel Martínez de la Escalera y Luis Báguena Corella describieron 
hasta 15 especies ibéricas y norteafricanas de los géneros Ceramida y Elaphocera cuyos tipos se conservan en las colecciones de Entomología del Museo Nacional de Ciencias Naturales de Madrid (MNCN). López-Colón ha revisado en sucesivos trabajos $(1992,1993,1994,1995 a)$ las series típicas de las especies de Ceramida descritas por estos autores, pero es necesaria una revisión similar en el caso del género Elaphocera, ya que en la mayoría de las especies no hay designación original del tipo.

En el marco de la investigación taxonómica de los géneros Elaphocera y Ceramida que viene desarrollando uno de los autores (I. Sanmartín), se revisan en este trabajo las series típicas de ocho especies de Elaphocera, a fin de aclarar y estabilizar el estatus nomenclatural de los siguientes taxones: Elaphocera (Elaphocerida) ibicensis Escalera, 1926; Elaphocerida segurensis Escalera, 1923; Elaphocerida pygmaea Escalera, 1923; Elaphocerida abdelkrimi Escalera, 1934; Elaphocera pardoi Escalera, 1931; E. (Elaphocerida) ampla Báguena, 1955; E. (Elaphocerida) torressalai Báguena, 1955; Elaphocera baguenae Mancini, 1926. Actualmente, sólo E. ibicensis, E. segurensis, E. ampla y E. baguenae se conservan como especies válidas (Baraud, 1992). El resto de las especies han sido o bien sinonimizadas, como es el caso de $E$. pygmaea $(=$ E. autumnalis Motschulsky, 1859$), E$. abdelkrimi (=E. sulcatula (Fairmaire, 1884)) y E. torressalai ( $=$ E. baguenae Mancini, 1926), o bien han cambiado de estatus taxonómico, como E. par$d o i$, ahora considerada como una subespecie de $E$. barbara Rambur, 1843.

Se designan también los lectotipos de las especies E. ampla, E. segurensis, E. pygmaea y E. torressalai.

\section{Elaphocerida pygmaea Escalera, 1923: 405}

Esta especie fue descrita por Escalera sobre una serie de 11 ejemplares machos procedentes de Sorbas (Almería). En la colección de entomología del MNCN se conservan 7 ejemplares de la serie típica. Todos ellos llevan la etiqueta blanca, manuscrita: "Sorbas".

Designamos como LECTOTIPO el ejemplar que se encuentra en mejor estado y que se ajusta mejor a la descripción original. Se le adjunta la etiqueta roja impresa de "Lectotipo. I. Sanmartín, des."; por detrás: "Elaphocerida pygmaea Escalera, 1923. Lectotipo. 1996 (des.)". Los otros 6 ejemplares pasan a ser etiquetados como Paralectotipos, con la etiqueta roja impresa de "Paralectotipo"; por detrás: I. Sanmartín, des. 1996. E. pygmaea".

Toda la serie típica tiene el número del Catálogo de Tipos de la colección de Entomología del MNCN: 8444.

ESTATUS ACTUAL: Esta especie ha sido sinonimizada con Elaphocera autumnalis Motschulsky, 1859 por Báguena (1955).

\section{Elaphocerida segurensis Escalera, 1923: 408}

La especie fue descrita sobre un número indeterminado de ejemplares recolectados en Elche de la Sierra (Albacete) en el sureste de España. En la colección del MNCN se encuentra una serie de 8 ejemplares, 7 machos y 1 hembra, de esta localidad, que concuerdan con la descripción dada por Escalera. López-Colón (1989) nombró estos 8 ejemplares como pertenecientes a la serie típica pero no designó el lectotipo, por lo que procedemos a ello.

LECTOTIPO: 1 ejemplar macho que lleva las siguientes etiquetas: "Elchecico 28-3-1916" (blanca, manuscrita) y "E. segurensis Esc. Typo" (blanca, manuscrita, de la mano del propio Escalera). Se le adjunta la etiqueta roja, impresa, de "Lectotipo. I. Sanmartín, des.”; por detrás manuscrita: "Elaphocera segurensis Escalera. Lectotipo. I. Sanmartín, 1996".

PARALECTOTIPOS: 4 ejemplares machos con la etiqueta: "Elchecico 28-3-1916"; 1 ejemplar macho con las etiquetas: "Elchecico, 28-3-1916" (blanca, manuscrita), "Elaphocera segurensa Esc" (blanca, manuscrita) y "Elaphocera segurensis. L. Báguena det." (blanca, manuscrita); 1 ejemplar macho con las etiquetas: "Elchecico, 28-3-1916" (blanca, manuscrita) y "Elaphocera segurensis. J.I. LópezColón, det. 1993" (blanca, manuscrita); 1 ejemplar hembra con la etiqueta: "Elchecico, 28-3-1916" (blanca, manuscrita). A estas etiquetas se les añaden las etiquetas rojas, impresas, de "Paralectotipo".

Como indican López-Colón \& Plaza-Lama (1989), "Elchecico" no corresponde a ningún término municipal, sino que es probablemente un apelativo de Elche de la Sierra. En su descripción original, Escalera nombra como localidad típica: "Elche de la Sierra (Murcia)"; sin embargo, esto debe ser un error del propio autor porque el municipio de Elche de la Sierra pertenece a la provincia de Albacete. 
Toda la serie típica tiene el número del Catálogo de Tipos de la colección de Entomología del MNCN: 8445.

EsTATUS ACTUAL: Esta especie debe conocerse como Elaphocera segurensis (Escalera, 1923) de acuerdo con Baraud (1992).

\section{Elaphocera (Elaphocerida) ibicensis Escalera, 1926: 160}

La especie fue descrita por Escalera sobre un único macho procedente de San Antonio (Ibiza) en las Islas Baleares. En el MNCN se conserva un ejemplar que concuerda con la detallada descripción original y lleva una etiqueta, escrita a mano por el propio Escalera, con la palabra "tipo". Al ser éste el único ejemplar nombrado por Escalera en la descripción original, debe considerarse como el HoLOTIPO por monotipia (ICZN, 1985). El ejemplar lleva las siguientes etiquetas: "S. Antonio. Ibiza. 41925" (blanca, manuscrita del propio Escalera) y "Elaphocerida ibicensis Esc. Tipo" (blanca, manuscrita de Escalera). A las que se añade una nueva etiqueta roja, impresa, de "Holotipo".

Este ejemplar tiene el número del Catálogo de Tipos de la colección de Entomología del MNCN: 8446.

Estatus ACTUAL: Esta especie debe conocerse como Elaphocera ibicensis Escalera, 1926 (Baraud, 1992).

\section{Elaphocera pardoi Escalera, 1931: 715}

Aunque en el trabajo original Escalera no designa expresamente el tipo, la especie fue descrita sobre un único macho del Gurugú (Melilla) (Anselmo Pardo, leg.), conservado en la colección de entomología del MNCN (ex col. Escalera). Este debe ser considerado como el HolotiPo por monotipia. Este ejemplar tiene las siguientes etiquetas: "Gurugú. 17-12-1931" (blanca, manuscrita, de la mano de Escalera) y "E. pardoi Esc. Typo" (blanca, manuscrita de Escalera). A las que se añade una nueva etiqueta roja, impresa, de "Holotipo".

Este ejemplar tiene el número del Catálogo de Tipos de la colección de Entomología del MNCN: 8450.

ESTATUS ACTUAL: Esta especie debe ser considerada como Elaphocera barbara pardoi Escalera, 1931, una subespecie de Elaphocera barbara Rambur, 1843, según Baraud (1979).

\section{Elaphocerida abdelkrimi Escalera, 1934: 129}

Escalera describe la especie sobre un único ejemplar macho procedente de la Bahía de Alhucemas (Pardo, leg.) que designa en su trabajo como tipo, por lo que se trata del Holotipo. Este ejemplar se conserva en la colección del MNCN junto con otro macho procedente de Ayin-Yil (Marruecos). El Holotipo lleva las etiquetas: "Axdir. 5-1933" (blanca, manuscrita, de la mano de Escalera) y "E. abdelkrimi Esc. Typo" (blanca, manuscrita de Escalera). A las que se añade una nueva etiqueta roja, impresa, de "Holotipo".

Este ejemplar tiene el número del Catálogo de Tipos de la colección de Entomología del MNCN: 8449.

ESTATUS ACTUAL: Elaphocerida abdelkrimi ha sido sinonimizada con Elaphocera sulcatula Fairmaire, 1884 por Baraud (1979).

\section{Elaphocera (Elaphocerida) ampla Báguena,} 1955: 147

Esta especie fue descrita por Báguena sobre 6 machos procedentes de Iznájar (Córdoba), que se conservan en el MNCN. Designamos como LeCTOTIPO el ejemplar que se encuentra en mejor estado y que lleva la etiqueta: "Iznájar (Córdoba). IV-1909. Exp. del Museo" (blanca, impresa). A este ejemplar se le añade la etiqueta roja, manuscrita, de "Lectotipo. I. Sanmartín, des.”; por detrás manuscrita: "Elaphocera ampla Baguena. Lectotipo. I. Sanmartín, des. 1996".

PARALeCtOTIPOS: 4 machos también con la etiqueta: "Iznájar (Córdoba). IV-1909. Exp. del Museo" (blanca, impresa); 1 macho con esa etiqueta y otra de la mano de nuestro colega J. I. LópezColón: "Elaphocera ampla Bág macho. J. I. LópezColón det, 1993" (blanca, manuscrita). A estos ejemplares se les añade una etiqueta roja, manuscrita, de "Paralectotipo".

Toda la serie típica tiene el número del Catálogo de Tipos de la colección de Entomología del MNCN: 8447.

Estatus aCtUAL: Esta especie debe conocerse como Elaphocera ampla Báguena, 1955 (Baraud, 1992).

\section{Elaphocera baguenae Mancini, 1926: 96}

Mancini describió la especie sobre 7 ejemplares machos recolectados por Báguena en El Plá, cerca 
de Manises, (Valencia) y le dedicó la especie al propio Báguena. Cinco de los ejemplares de la serie típica se conservan en la colección A. Dodero y los otros dos en la colección del propio Mancini, ambas en el Museo civico di Storia Naturale de Génova.

En la colección de entomología del MNCN se conservan 6 ejemplares: 2 ejemplares machos etiquetados de "El Plá, Valencia. Col. Báguena" (etiqueta blanca impresa); 2 ejemplares machos etiquetados de "El Plá, Valencia. Moroder" (etiqueta blanca manuscrita), 1 ejemplar hembra etiquetado de "Villamarchante (Valencia). Moroder" (etiqueta blanca impresa) y 1 ejemplar macho etiquetado de "Paterna (Valencia). Col. Báguena" (etiqueta blanca impresa). Los cinco primeros ejemplares han sido considerados erróneamente como pertenecientes a la serie típica (López-Colón, 1995b). Esto se debe, por un lado, a que los dos ejemplares de El Plá de la col. Báguena son locotípicos y, probablemente, pertenecen a la misma serie de ejemplares de donde proviene la serie típica. Por otro lado, Baraud (1992), al citar equivocadamente Villamarchante como segunda localidad típica, indujo al error de considerar el ejemplar de Villamarchante del MNCN como perteneciente a la serie típica. Este error ya ha sido enmendado por el propio López-Colón (1997).

ESTATUS ACTUAL: Esta especie debe conocerse como Elaphocera baguenae Mancini, 1926. Posterior a su descripción, la terminación del nombre específico fue modificada como baguenai y la especie pasó a llamarse E. baguenai (Báguena, 1955; Baraud, 1992, López-Colón, 1995). Sin embargo, esta enmienda no está justificada ya que el nombre original es válido según el artículo 31(a) (i) del ICZN (1985).

\section{Elaphocera (Elaphocerida) torressalai Báguena,} 1955: 143

Esta especie fue descrita sobre un número indeterminado de ejemplares de Torrente, Alcira, Játiva (Valencia), y Pego (Alicante). En la ex. col. Báguena del MNCN se conservan 3 ejemplares de la serie típica, de las tres primeras localidades nombradas. Como López-Colón menciona en su estudio de la especie (1995b), estos ejemplares están etiquetados como 1 holotipo y 2 cotipos. Según hemos comprobado, esta designación nunca fue publicada por lo que no sería válida y designamos aquí el lectotipo. El ejemplar previamente etiqueta- do como holotipo (de Torrente) no presenta el abdomen ni las cuatro patas posteriores por lo que preferimos designar como lectotipo al ejemplar de Játiva. Este se encuentra en buen estado de conservación y concuerda con la descripción original que da para la especie un color rojo pajizo.

LECTOTIPO: Ejemplar macho de color rojo pajizo con las siguientes etiquetas: "Elaphocera boscai Per. Játiva. Boscá!" (blanca, manuscrita), "E (Elaphocerida) torressalai mihi. Det. Dr. L. Báguena" (blanca, manuscrita) y "Cotipo" (roja, impresa). A estas etiquetas se les añade una nueva etiqueta roja, impresa, de "Lectotipo". I. Sanmartín, des."; por detrás manuscrita: Elaphocera torressalai Báguena. I. Sanmartin, 1996”.

PARALECTOTIPOS: 1 ejemplar macho intacto de pronoto y cabeza oscuros con las etiquetas: "Alcira. Valencia. Col. Báguena" (blanca, impresa), "E (Elaphocerida) torressalai mihi. Det. Dr. L. Báguena" (blanca, manuscrita) y "Cotipo" (roja, impresa); 1 ejemplar macho rojo pajizo que carece de abdomen con las etiquetas: "Torrente. Valencia. Col. Báguena", " E (Elaphocerida) torressalai mihi. Det. Dr. L. Báguena" (blanca, manuscrita) y "Holotipo" (roja, impresa). A estos ejemplares se le coloca una nueva etiqueta roja, impresa, de "Paralectotipo".

Toda la serie típica tiene el número del Catálogo de Tipos de la colección de Entomología del MNCN: 8448.

ESTATUS ACTUAL: Esta especie ha sido sinonimizada con Elaphocera baguenae Mancini, 1926 por López-Colón (1995b). El nombre específico original torres-salai fue corregido por el de torressalai a partir de Baraud (1977). De acuerdo con el ICZN, sólo se admite la presencia de un guión en el nombre específico cuando la primera palabra antes del guión es una sóla letra (ICZN, 1985, art. 31 (d)).

\section{AGRADECIMIENTOS}

Queremos agradecer a J. I. López-Colón su ayuda en la búsqueda bibliográfica. Las conservadoras del Museo Nacional de Ciencas Naturales nos prestaron su inestimable y habitual colaboración. Trabajo financiado por el proyecto Fauna Ibérica III (PB89-0121).

\section{Referencias}

BÁGUENA, L., 1955. Observaciones sobre las especies ibéricas de Elaphocera Gené. Eos, 31(1-2): 123-153.

BÁGUENA, L., 1967. Scarabaeoidea de la Fauna íberobalear y pirenaica. Consejo Superior de Investigaciones Científicas (CSIC). Madrid. 576 pp. 
BARAUD, J., 1966. Le genre Elaphocera Gené: description de quatre nouvelles espéces d'Espagne. Eos, 42: 27-39.

BARAUD, J., 1975. Révision des espèces ibériques du genre Elaphocera Gené. Nouv. Rev. Entomol., V: 57-65.

BARAud, J., 1977. Coléoptères Scarabaeoidea, Faune de l'Europe occidentale: Belgique, France, GrandeBretagne, Italie, Péninsule Ibérique. Suppl. Nouv. Rev. Entomol., 7: 1-352 pp.

BARAud, J., 1979. Coléoptéres Scarabaeoidea de l'Afrique du Nord. $3^{\mathrm{a}}$ Note: Les Pachydemini. Ann. Soc. Entomol. Fr. (N. S.), 15(2): 247-310.

BARAud, J., 1987. Révision des Elaphocera d'Europe (Coléoptères, Melolonthidae). Ann. Soc. Entomol. Fr. (N. S.), 23(2): 125-134.

BARAud, J., 1992. Faune de France. Coléopteres Scarabaeoidea d'Europe. Société Linnéenne de Lyon y Fédération Française des Sociétés de Sciences Naturelles. Lyon. 856 pp.

Burmeister, C.H.C., 1855. Handbuch der Entomologie. IV, (2). Th. Enslin ed. Berlin.

DejeAn, DE M. LE CoMte, 1836. Catalogue des Coléoptères de la collection de M. le comte Dejean. $3^{\mathrm{a}}$ édition. Fascicles 1-4 (1-384 pp.). MequignonMarvis. Paris. 503 pp.

Dalla-Torre, K.W. VON., 1912-1913. Scarabaeidae, Melolonthinae. In: S. Schenkling (ed.). Coleopterorum Catalogus, pars 50. W, Junk Verlag. Berlin: 305-308.

EsCALERA, DE LA. M.M., 1923. Especies nuevas de Elaphocera (Col. Scarab.) de España. Bol. R. Soc. Esp. Hist. Nat., 23: 400-410.

ESCALERA, DE LA. M.M., 1926. Una Elaphocera Gené nueva de Baleares. Bol. R. Soc. Esp. Hist. Nat., 26: 160-162.

EsCALERA, DE LA. M.M., 1931. Una nueva especie de Elaphocera Gené de Marruecos (Col. Scarab). Bol. R. Soc. Esp. Hist. Nat., 31: 715-717.

EsCAlERA, DE LA. M.M., 1934. Una Elaphocerida Reitter nueva del Rif (Col. Scarab). Bol. R. Soc. Esp. Hist. Nat., 34: 129-131.

GENÉ, C.G., 1836. [Dix-sept Coléoptères nouveaux de Sardaigne]. Ann. Soc. Entomol. Fr., 5, Bull. (1): III.

Gené, C.G., 1837a. De quibusdam Insectis Sardiniae novis aut minus cognitis. Ins. Sardin., I: 28-30. 39 pp.

GENÉ, C.G., 1837b. De quibusdam Insectis Sardiniae novis aut minus cognitis. Mem. Accad. Torino, 39: 161-199.

INTERNATIONAL COMMISSION ON THE ZOOLOGICAL NOMENCLATURE (ICZN), 1985. International Code of Zoological Nomenclature, Third edition, adopted by the XX General Assembly of the International Union of Biological Sciences. International Trust for Zoological Nomenclature, London, XX + 336 pp.

JaCQuelin du VAL, C., 1860. Genera des Coléoptères d'Europe. Manuel entomollogique, III. A. Deyrolle, Paris. 463 pp.
KraAtZ, G., 1882. Kurze Revision der ElaphoceraArten. Deutsche Entomol. Z., 26: 17-31.

LAtreille, P.A., 1809. Genera Crustaceorum et Insectorum secundum ordinem naturalem in familias disposita, iconibus exemplisque plurimis explicata, 4. Pariis et Argentorat. König. 399 pp.

LóPEZ-Colón, J.I., 1989. Descripción de Elaphocerida ferreri, nueva especie anadaluza y algunos datos sobre especies del género Elaphocerida Reitter, 1901 de la Península Ibérica (Coleoptera, Scarabaeoidea). Nouv. Rev. Entomol. (N. S.), 6(2): 193-198.

LÓPEZ-ColÓN, J.I., 1992. Revisión de algunos tipos de especies endémicas de la Península Ibérica del género Ceramida Baraud, 1987 (Coleoptera: Scarabaeidae: Melolonthinae: Pachydemini). G. it. Entomol., 6: 229-233.

LOPEZ-ColÓN, J.I., 1993. Rehabilitación de Elaphocera bedeaui var. Adusta Kraatz, 1882 y su identidad con Elaphocera abderramani Escalera, 1923. Designación del lectotipo de Ceramida adusta (Kraatz, 1882) (Coleoptera: Scarabaeidae: Melolonthinae: Pachydemini). Nouv. Rev. Entomol. (N. S.), 10: 303-304.

LóPEZ-ColóN, J.I., 1994. Révision de quelques types du genre Ceramida Baraud, 1987 des collections d'Espagne (Coleoptera, Scarabaeidae, Melolonthinae, Pachydemini). L'Entomologiste, 50(2): 147-152.

LóPEZ-Colón, J.I., 1995a. Désignation du Lectotype d'Elaphocera bedeaui Erichson, 1840 (Col. Scarabaeidae, Melolonthinae). L'Entomologiste, 51(3): 131

LÓPEZ-ColóN, J.I., 1995b. Nueva sinonimia de Elaphocera ibérica (Coleoptera, Scarabaeidae). Nouv. Rev. Entomol. (N. S.), 12(2-3): 139-144.

LóPEZ-Colón, J.I., 1997. Errata y enmendata (Coleoptera, Scarabaeidae, Melolonthinae). Bol. Soc. Entomol. Aragon., 19: 62.

MadGe, R.B., 1988. The publication dates of Dejean's catalogues. Arch. Nat. Hist., 15(3): 317-321.

MAncini, C., 1926. Su alcuni Scarabeidi d'Italia e Spagna. Boll. Soc. Entomol. Ital., Génova, 58: 92-96.

Medvedev, S.I., 1952. Fauna S. S. S. R., Coleoptera, Scarabaeidae Melolonthidae. Moscow. Tome X. fasc. 2. $275 \mathrm{pp}$.

RAmbur, P., 1843. Monographie du genre Elaphocera. Ann. Soc. Entomol. Fr., 1(2): 329-358.

Reitter, E., 1902. Bestimmungs-Tabelle der Melolonthidae: Pachydemini, Sericini und Melolonthini. Verh. Nat. Ver. Brünn. XL: 95-105.

Recibido, el 8-I-1998 Aceptado, el 16-XI-1998 Publicado, el 31-XII-1998 